\title{
Meta-analisi del ruolo dell'attività fisica nel trat- tamento della stitichezza
}

\author{
Thomas Weiser, PhD, Sabine Landes, PhD
}

\begin{abstract}
Le attuali linee guida raccomandano l'introduzione di cambiamenti nello stile di vita (tra cui più attività fisica) come prima opzione per il trattamento della stitichezza. Questa metaanalisi ha esaminato gli effetti dell'attività fisica sui sintomi dei pazienti con stitichezza cronica. Circa la metà di coloro che hanno seguito un programma di allenamento della durata di diverse settimane ha sperimentato un effetto positivo sui sintomi. Negli studi clinici condotti con i lassativi bisacodile e sodio picosolfato, generalmente questi farmaci si sono dimostrati in grado di alleviare i sintomi già il primo giorno di trattamento.
\end{abstract}

\begin{abstract}
a stitichezza è un disturbo che può compromettere in
Lmaniera significativa la qualità della vita di coloro che ne soffrono [4], oltre ad avere implicazioni economiche considerevoli (per esempio in termini di visite dal medico e permessi per malattia). È quindi estremamente importante individuarne un trattamento che produca buoni risultati.
\end{abstract}

Le linee guida sul trattamento della stitichezza (cronica) propongono un algoritmo di trattamento articolato in diverse fasi. La prima fase prevede l'introduzione di cambiamenti nello stile di vita dei soggetti interessati (più attività fisica, aumento dell'assunzione di liquidi e fibre), la seconda l'utilizzo di lassativi da banco quali bisacodile, sodio picosolfato e macrogol, mentre la terza si basa sull'uso esclusivo di prodotti soggetti a prescrizione medica $[1,4]$.

Sono numerosi gli studi che hanno esaminato l'impatto dell'attività fisica sulla digestione nei pazienti che soffrono di stitichezza; i risultati di questi studi sono stati sintetizzati in una meta-analisi a opera di Gao et al. [2]. Gli autori hanno identificato 9 studi (per un totale di 680 pazienti) che hanno esaminato gli effetti dell'attività fisica sulla stitichezza. I soggetti nei gruppi di controllo si erano sottoposti ad agopuntura auricolare, avevano assunto compresse di Ma Zi Ren (medicina tradizionale cinese), avevano ricevuto informazioni specifiche sulla stitichezza oppure nessun trattamento particolare. Le attività sportive erano estremamente varie, ma si trattava prevalentemente di attività aerobiche, quali Qi Gong, Baduanjin (entrambe discipline di origine asiatica) o camminata (veloce); solo uno studio ha esaminato gli effetti dell'allenamento di rinforzo muscolare.

\begin{abstract}
Lallenamento prevedeva da 60 a 420 minuti di attività fisica a settimana per un periodo compreso tra 4 e 24 settimane. Il parametro scelto per valutare gli effetti dell'attività fisica è stato il rischio relativo (RR) di sollievo dai sintomi della stitichezza. Che cosè emerso dall'analisi?
\end{abstract}

Nonostante la natura estremamente eterogenea dell'attività fisica praticata, il risultato è stato comunque piuttosto chiaro: i pazienti che hanno completato il programma di allenamento hanno sperimentato un maggiore sollievo dai sintomi della stitichezza rispetto ai pazienti nei gruppi di controllo. Tuttavia, quest'effetto è stato osservato solo dopo l'allenamento aerobico (RR pari a 2,42 a favore dei gruppi che hanno seguito un programma di allenamento). L'allenamento anaerobico, invece, non ha prodotto alcun effetto (RR pari a 0,85 ). La combinazione delle due forme di allenamento ha prodotto un RR pari a 1,97. Pertanto, l'attività fisica sembra essere utile per dare sollievo dai sintomi della stitichezza, e i risultati migliori si ottengono con almeno 140 minuti a settimana di allenamento aerobico. Alcuni studi hanno esaminato anche parametri quali vitalità, benessere e qualità della vita dei pazienti; anche in questo caso, l'allenamento ha avuto un effetto positivo.

Quali sono dunque le implicazioni di questi risultati per la pratica clinica quotidiana? Qual è il significato dei RR misurati? Prendiamo un esempio: su 10 pazienti che soffrono di stitichezza, solo 5 trarranno giovamento dall'attività fisica, anche avendola praticata per un periodo compreso tra 4 e 24 settimane (vedere la figura 1, riquadro a sinistra).

Evid Self Med 2021;1:210047 | https://doi.org/10.52778/efsm.21.0047

Affiliazione/Corrispondenza: Thomas Weiser, PhD, Sabine Landes, PhD, Consumer Healthcare Medical Affairs, Sanofi-Aventis Deutschland GmbH, Industriepark Hoechst, 65026 Frankfurt am Main, Germany (thomas.weiser@sanofi.com) 


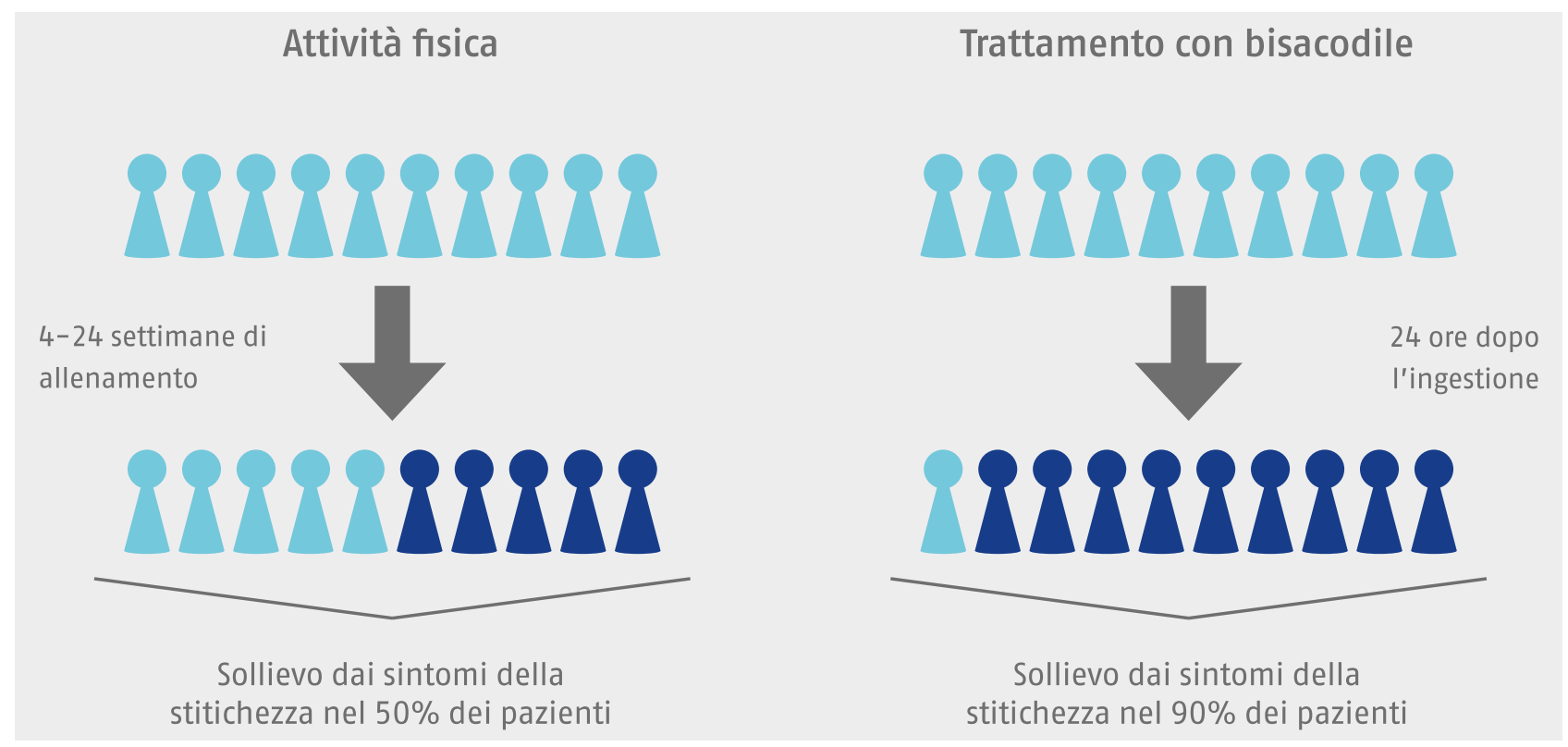

A sinistra: L'allenamento per un periodo compreso tra 4 e 24 settimane produce un sollievo dai sintomi della stitichezza in circa il $50 \%$ dei soggetti. A destra: Il trattamento con bisacodile stimola l'evacuazione entro 24 ore in circa il $90 \%$ dei pazienti trattati.

Secondo le attuali linee guida $[1,4]$, la prima fase del trattamento della stitichezza prevede l'introduzione di cambiamenti nello stile di vita (attività fisica, idratazione, assunzione di fibre); se queste misure non funzionano, si passa ai lassativi (per esempio macrogol, bisacodile, sodio picosolfato). Due studi clinici nei quali bisacodile e sodio picosolfato sono stati messi a confronto con un placebo hanno dimostrato efficacia, sicurezza e tollerabilità di entrambi questi farmaci per il trattamento della stitichezza. Per esempio, circa il 70\% dei pazienti trattati con sodio picosolfato e il $90 \%$ dei pazienti che hanno utilizzato bisacodile sono riusciti ad evacuare nelle prime 24 ore dall'ingestione [3, 5 dati in archivio, Sanofi Aventis Deutschland GmbH; figura 1, riquadro a destra], e il sollievo dai sintomi della stitichezza è stato osservato per l'intera durata dello studio (4 settimane in entrambi i casi).

Conclusioni: Secondo Goa [2], l'attività fisica praticata per diverse settimane ha un effetto positivo sulla stitichezza in circa la metà dei soggetti. Solitamente i lassativi contenenti bisacodile e sodio picosolfato offrono sollievo dai sintomi nelle prime 24 ore dall'ingestione fino al $90 \%$ dei pazienti.

\section{Letteratura}

1. Andresen et al. S2k Guideline for Chronic Constipation: Definition, Pathophysiology, Diagnosis and Therapy. Z Gastroenterol. 2013;51:651-672.

2. Gao R, Tao Y et al. Exercise Therapy in Patients with Constipation: A Systemic Review and Meta-Analysis of Randomized Controlled Trials. Scandinavian Journal of Gastroenterology. 2019;54:169-177.

3. Kamm MA, Müller-Lissner S et al. Oral Bisacodyl is Effective and Well-Tolerated in Patients with Chronic Constipation. Clinical Gastroenterology and Hepatology. 2011;9:577-83.

4. McCormick D. Managing Costs and Care for Chronic Idiopathic Constipation. The American Journal of Managed Care. 2019;25:S63S69.

5. Müller-Lissner S, Kamm MA et al. Multicenter, 4-Week, DoubleBlind, Randomized, Placebo-Controlled Trial of Sodium Picosulfate in Patients With Chronic Constipation; American Journal of Gastroenterology. 2010;105:897-903.

Conflitto di interessi: T. Weiser e S. Landes sono dipendenti di Sanofi.

Dichiarazioni: Pubblicazione finanziata da Sanofi Aventis Deutschland $\mathrm{GmbH}$.

Informazioni sul manoscritto

Inoltrato il; 14.09 .2020

Accettato il: 27.12 .2020

Pubblicato il: 16.08.2021 